\title{
МОДЕЛИРОВАНИЕ МЕХАНИЧЕСКИХ ПРОЦЕССОВ В ПЛОСКОСТНЫХ МОДЕЛЯХ КОНСТРУКЦИЙ РЭС
}

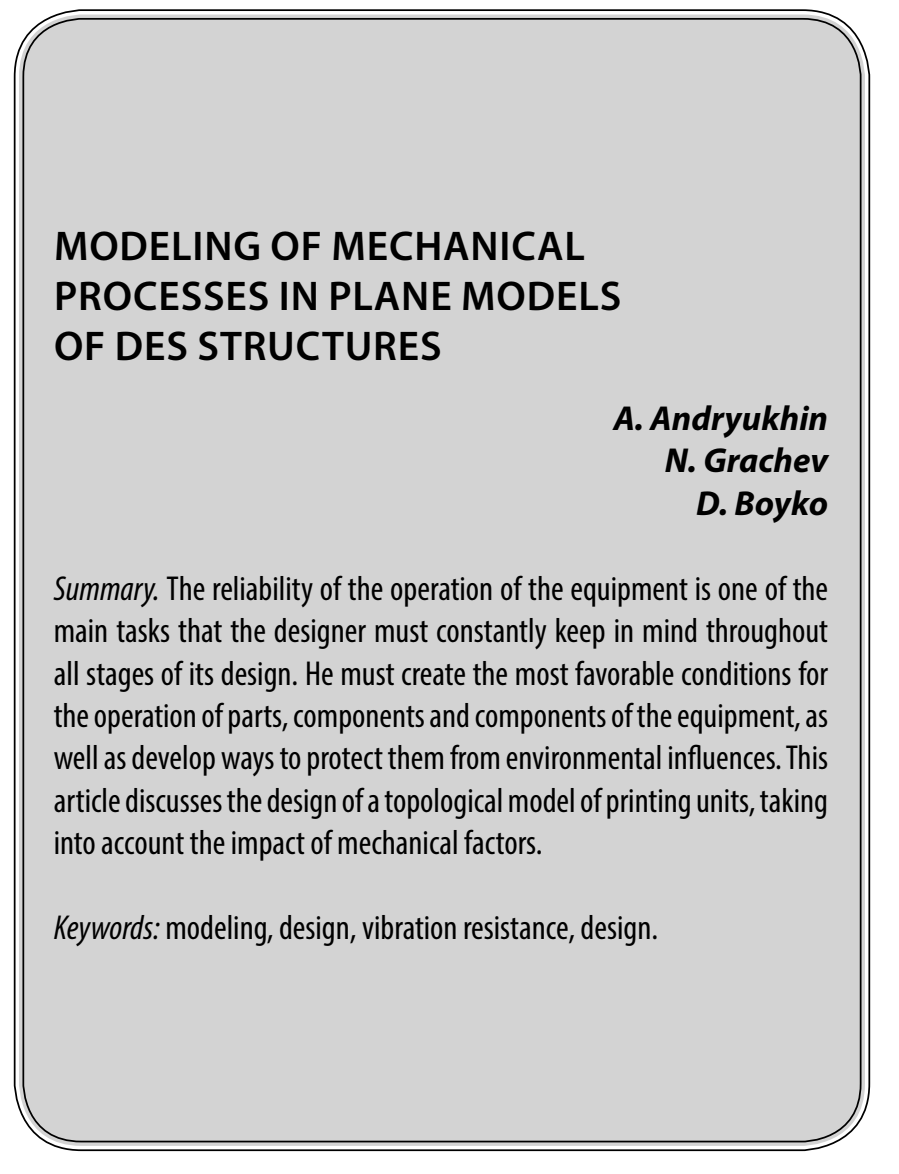

B ибрационные воздействия, действующие на аппаратуру, охватывают широкий интервал частот от 5 до 5000 Гц. Ударные и вибрационные нагрузки ухудшают надёжность РЭС, поэтому предпринимаются попытки тем или иным способом учесть влияние механических воздействий на надёжность. Около $50 \%$ отказов радиоэлектронных средств возникает из-за недопустимо больших деформаций монтажных плат, разгерметизации соединений корпусных элементов, нарушений в электромонтаже, разрушение паянных и сварных соединений при механических и термических воздействиях. В связи с этим конструкторам и технологам РЭС и её элементарной базы приходится решать сложные задачи обеспечения прочности различных по структуре и свойствам узлов.

Исходным аналитическим выражением, описывающим механические колебания плоской конструкции (печатного узла), в рамках технической теории тонких пластин является бигармоническое уравнение [1]:
Андрюхин Александр Гавриилович

К.т.н., дочент, МИРЭА - Российский технологический университет (г. Москва)

pr1110@list.ru

Грачев Николай Николаевич

К.т.н., профессор, МИРЭА - Российский технологический университет (г. Москва)

nngrachev@mail.ru

Бойко Денис Владимирович

МИРЭА - Российский технологический университет,

2. Москва

den87-2004@mail.ru

Аннотация. Безотказность функционирования аппаратуры является одной из основных задач, которую постоянно должен иметь в виду конструктор на протяжении всех этапов её проектирования. Он должен создать наиболее благоприятные условия для работы деталей, элементов и узлов аппаратуры, а также разработать способы их защиты от воздействия окружающей среды. В данной статье рассматривается проектирование топологических модели печатных узлов с учетом воздействия механических факторов.

Ключевые слова: моделирование, проектирование, вибропрочность, конструкция.

$$
\begin{aligned}
& \overline{D_{1}}=\left(\frac{\partial^{4} W}{\partial X^{4}}\right)+2 \bar{D}_{3}\left(\frac{\partial^{4} W_{1}}{\partial x^{2} \partial y^{2}}\right)+\overline{D_{2}}\left(\frac{\partial^{4} W_{1}}{\partial y^{4}}\right)+ \\
& +m_{1}\left(\frac{\partial^{2} W_{1}}{\partial t^{2}}\right)=0 .
\end{aligned}
$$

Где $w_{l}$ - прогиб печатной платы в точке с координатами х, у в момент времени $t$;

$m_{1}$ - масса печатного узла, приходящаяся на единицу площади в точке 1 ;

$$
\overline{D_{1}}=\frac{\overline{E_{1}} S^{3}}{12\left(1-\mu_{1} \mu_{2}\right)}-
$$

цилиндрическая жесткость печатней платы по оси х;

$$
\overline{D_{1}}=\frac{\overline{E_{1}} S^{3}}{12\left(1-\mu_{1} \mu_{2}\right)}-
$$

цилиндрическая жесткость печатной платы по оси у.

$\overline{D_{3}}=\overline{D_{1}} \mu_{2}+2 \overline{D_{m}}=\overline{D_{2}} \mu_{1}+2 \overline{D_{k}}$ 


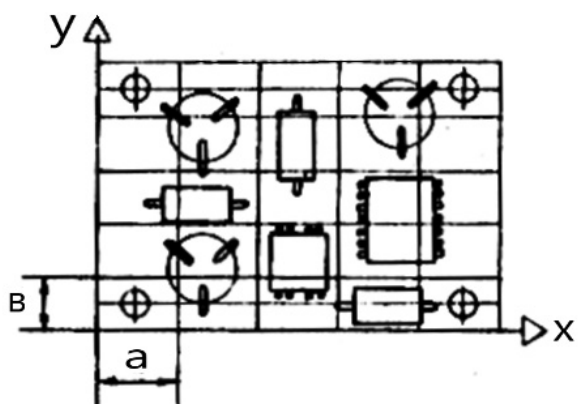

Рис. 1. Чертеж печатного узла с нанесенной сеткой

главная жесткость;

$\bar{D}_{k}=\frac{\bar{G} S^{3}}{12}$

жесткость кручения;

$\bar{G}=\frac{\overline{E_{45}}}{2\left(1+\mu_{45}\right)}-$

модуль сдвига материала печатной платы;

$S$ - Толщина платы;

$E_{1}, E_{2}, E_{45}$ - модули упругости материала платы по осям х, у и под углом $45^{\circ}$ к осям соответственно;

$\mu_{1}, \mu_{2}, \mu_{45}$ - коэффициенты Пуансона материала плоты по осям х, у и под углом $45^{\circ}$ к осям соответственно (считаем, что направление осей $\mathrm{x}$ и у совмещено с направлением сторон печатной платы).

Так как на печатной плате установлены ЭРЭ, необходимо учесть их массу и цилиндрическую жесткость, которая вычисляется по тем же формулам, что и для печатной платы. Это относится в основном к ЭРЭ, жестко скрепленным с платой. Для навесных ЭРЭ их цилиндрической жёсткостью можно пренебречь. Вычисленную жесткость ЭРЭ суммируем с цилиндрической жесткостью соответствующего участка ПУ, то есть того, где установлен ЭРЭ.

Для учета рассеяния энергии на внутреннее трение в материале платы используется гипотеза Сорокина, сущность которой состоит в комплексном представлении упругих постоянных материала. Комплекс ный модуль упругости выразится как:

$$
\overline{E_{1}}=E_{1}+(i+j \gamma), i=1,2,45 \ldots
$$

Где $E_{l}$ - статический модуль упругости;

$\gamma$-коэффициент механических потерь.

Коэффициент механических потерь связан с логарифмическим дек рементом затухания колебаний (ЛДЗК) следующим соотношением:

$$
\gamma=\Lambda / k
$$

Значения ЛДЗК в зависимости от механических напряжений при изгибных колебаниях для ряда конструкционных материалов, применяемых для из готовления несущих конструкций РЭС, приведены в [2].

Для определения $ү$ необходимо знать механическое напряжение, которое можно получить только после решения уравнения (1). Поэтому решение приходится проводить в несколько итераций. Вначале решается уравнение при ориентировочном значении $\gamma$, затем вычисляется напряжение и корректируется ү.

При проектировании конструкций РЭС большое значение имеет ана лиз их динамических свойств в частотной области [3]. Для перехода в (1) в частотную область воспользуемся экспоненциальной формой представления переменных величин, в данном случае прогиба w1;

$$
W_{t}^{(t)}=\overline{W_{t}} \theta^{j \varpi t},
$$

Где $\bar{W}$ - комплексная амплитуда прогиба в точке 1 печатного узла.

Замерим в (1) частные производные по координатам приближенными выражениями через конечные разности:

$$
\begin{aligned}
& \frac{\partial^{4} W_{1}}{\partial x^{4}}=\left(i \in W_{1}-4\left(W_{1}+W_{3}\right) W_{10}+W_{12} 1 / a^{4}\right. \\
& \frac{\partial^{4} W_{1}}{\partial y^{4}}=\left(i \in W_{1}-4\left(W_{1}+W_{4}\right) W_{9}+W_{11} 1 / b^{4}\right. \\
& \frac{\partial^{4} W_{1}}{\partial x^{4} \partial y^{2}}=\left(4 W_{1}-2\left(W_{1}+W_{2}+W_{3}+W_{4}\right)+\right. \\
& \left.W_{5}+W_{6}+W_{7}+W_{8}\right) / a^{2} b^{2}
\end{aligned}
$$

Где $a$ и $b$ - шаги сетки по осям X и Ү, накладываемой на чертеж печатного узла, как это показано на рисунке 1;

$W_{1}-W_{12}$ - прогибы печатного узла от исходного положения равновесия в точках 1-12, расположенных в уз- 


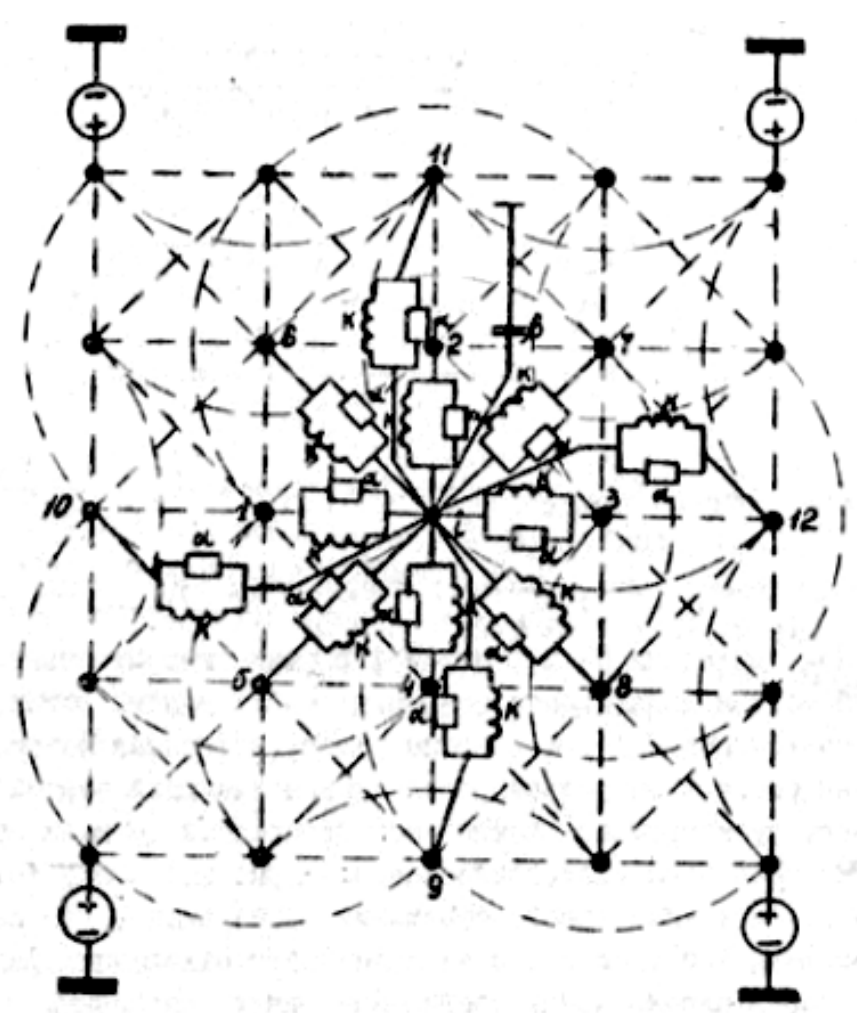

Рис. 2. Топологическая модель печатного узла РЭС

лах сетки вокруг произвольной точки 1, как это показано на рисунке 2.

Подставим (4) и (5) в (1). После группировки слагаемых с одинаковыми значениями прогибов получим:

$$
\begin{aligned}
& -4 \frac{\overline{D_{1}} b^{2}+\overline{D_{3}} a^{2}}{a^{4} b^{2}} \sum_{i=1,3}\left(\bar{W}-\overline{W_{1}}\right)- \\
& -4 \frac{\overline{D_{2}} a^{2}+\overline{D_{3}} b^{2}}{a^{2} b^{4}} \sum_{i=2,4}\left(\bar{W}_{i}-\overline{W_{t}}\right)+ \\
& +\frac{2 \overline{D_{3}}}{a^{2} b^{3}} \sum_{r=s}\left(\bar{W}_{a}-\overline{W_{1}}\right)+ \\
& \left.+\frac{\overline{D_{1}}}{a^{4}} \sum_{i=10 ; 12} \overline{\left(W_{1}\right.}-\overline{W_{1}}\right) \frac{\bar{D}_{2}}{b^{4}} \sum_{i=10 ; 12}\left(\overline{W_{1}}-\overline{W_{1}}\right)+ \\
& \left.+\frac{\overline{D_{2}}}{b^{4}} \sum_{(W}-\bar{W}_{1}\right)+m_{t}(j \varpi)^{a} \bar{W}_{1}=0,
\end{aligned}
$$

Поскольку чертеж на рис. 1 разделен сеткой на участки площадью ab каждый, то в последнем слагаемом полученного уравнения целесообразно перейти к массе участка, в центре которого расположена точка 1. Разде-

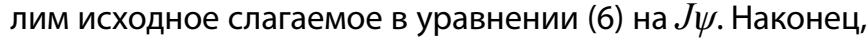
раскроем в уравнении (1) комплексной жесткости в виде:

$$
\overline{D_{1}}=D_{1}(i+j \gamma), i=1,2,3
$$

и выделим отдельно консервативные и диссипативные параметры. После указанных преобразований уравнение (1) примет вид, который можно представить в унифицированных обозначениях:

$$
\sum_{i=1}^{12} h_{1}\left(\varphi_{1}-\varphi_{1}\right)+h_{1,0} \varphi_{1}=0 .
$$

Где $\varphi_{1}=\bar{W}_{1}, \varphi_{1}=\bar{W}_{1}$ - потенциальные переменные величины узлов топологической модели печатного узла;

Обобщённые параметры ветвей модели выразим уравнением:

$$
h_{1, t}=\frac{1}{j \varpi k_{1,1}}+a_{1,2} h_{1,0}=j \varpi \mathrm{B}_{1,0} .
$$

Причем:

$$
\begin{aligned}
& k_{1}=k_{1,3}=-\frac{a^{3} b}{4\left(D_{1} b^{2}+D_{3} a^{2}\right.} ; \\
& k_{1,2}=k_{1,4}=-\frac{a^{3} b}{4\left(D_{2} a^{2}+D_{3} b^{2}\right)} ;
\end{aligned}
$$




$$
\begin{aligned}
& k_{1}=-\frac{a b}{2 D_{3}} ; r=5,6,7,8 ; k_{1,9}=k_{1, t}=\frac{b_{3}}{D_{2} a} ; \\
& k_{1,10}=k_{1,12}=\frac{a^{3}}{D_{t} b} ; \\
& a_{1,2}=\frac{\Gamma}{\varpi k_{1,2}} r=1 \ldots .12 ; \mathrm{B}_{1,0}=a b m_{1}
\end{aligned}
$$

Как видно из (7), любой 1-й узел синтезированной топологической модели должен быть соединен с 12 узлами сетки, окружающими 1-й узел (см. рис. 2), и с общим узлом, потенциал которого принят за нуль (на рис. 2 он условно обозначен полужирной чертой). На рис. 2 показаны пунктиром все ветви тог логической модели прямоугольного участка печатного узла, показанного на рис. 1 между точками крепления 1, 2, 3 и 4. Из первого выражения (8) видно, что каждая ветвь, соединяющая 1-й узел с гнездом из двенадцати выделенных узлов, представляет собой параллельное соединение двух пассивных компонентов: консервативного с параметром к; и диссипативного с параметром. На рис. 2 показано электрическое изображение этих компонентов, что подчеркивает возможность построения топологической механической модели ПУ в виде электрической сетки. Ка общий узел из каждого 1-го узла модели идет ветвь, содержащая консервативный компонент с параметром $\beta 1$. Потенциальные переменные узлов, соответствующих мостам крепления платы, должны быть заданы. Поэтому ветви этих узлов, идущие на общий узел, содержат потенциальные активные компоненты.

В результате расчета могут быть получены перемещение и ускорение в узлах модели печатного узла. Механические напряжения по оси х в 1-й точке рассчитываются по формуле:

$$
\left.\mathrm{y}_{1}=\frac{E_{1} S}{2\left(1-\mathrm{M}_{1} \mathrm{M}_{a}\right)}\left(\frac{\partial^{2} W_{1}}{\partial y^{2}}\right)+\mathrm{M}_{1} \frac{\partial^{2} W_{1}}{\partial y^{2}}\right),
$$

и по оси у:

$$
\left.\mathrm{y}_{1}=\frac{E_{1} S}{2\left(1-\mathrm{M}_{1} \mathrm{M}_{a}\right)}\left(\frac{\partial^{2} W_{1}}{\partial y^{2}}\right)+\mathrm{M}_{1} \frac{\partial^{2} W_{1}}{\partial y^{2}}\right) .
$$

Эквивалентное механическое напряжение в узле модели ПУ опреде ляется согласно энергетической гипотезе по формуле:

$$
\mathrm{y}=\sqrt{\mathrm{y}_{1}^{2}+\mathrm{y}_{2}^{2}-\mathrm{y}_{1} \mathrm{y}_{2}} .
$$

Выражения (9), (10) аналогично (5) представляются конечными разностями. Напряжение б используется для коррекции $\gamma$.

Для анализа механических процессов в ЭРЭ необходимо иметь возможность определять перемещение, ускорение и угол изгиба в любой точке ПУ по осям $\mathrm{X}$ и Ү. Нужно перейти от дискретной модели к непрерывной путем интерполяции функции двух переменных $\mathrm{x}$ и у по четырем точкам:

$$
\begin{aligned}
& W(x, y)=(i-p)(i-m) W_{i, j} \\
& +p(i-m) W_{i+i, j}+(i-p) m w_{i, j+1}+p m w_{i-1, j+1}
\end{aligned}
$$

Где $p=(x-x l) / h, m=(y-y) / 1 ; h$ - шаг изменения $x ; I-$ шаг изменения $y$;

$$
W_{i, j} ; W_{i+i, j} ; W_{i, j+i} ; W_{i+i, j+t}-
$$

перемещения в узлах дискретной модели.

Учитывая, что угол изгиба платы вдоль определенной оси - это есть первая производная функции перемещения по соответствующей координате, получим на основе (12) выражения для углов изгиба:

$$
\begin{aligned}
& \mathrm{и}_{x}=\left(1 / \varpi^{2}\right)\left(\left(W_{1=1, j}-W_{i, j}\right) / h+\left(W_{1, j}-W_{i+i, j}-\right.\right. \\
& \left.\left.W_{i, j+i}+W_{i+i, j=1}\right)\left(y-y_{1}\right) / h l\right), \\
& \mathrm{и}_{y}=\left(1 / \varpi^{2}\right)\left(\left(W_{1=1, j}-W_{i, j}\right) / 1+\left(W_{1, j}-W_{i+i, j}-\right.\right. \\
& \left.\left.W_{i, j+i}+W_{i+i, j=1}\right)\left(x-x_{1}\right) / h l\right) .
\end{aligned}
$$

Где и $x$, и $y$-углы изгиба сечений ПУ вдоль осей $\mathrm{X}$ и Ү соответственно.

Расчет ПУ аналитическими методами для ограниченного числа вариантов крепления плат с равномерным расположением ЭРЭ представлен в [4]. Там нет необходимости в дискретизации и интерполяции, так как по полученной формуле можно определить значение механической характеристики в любой точке. При этом в [4] не учитывается анизотропия свойств печатной платы по различным направлениям, а также используется линейная механическая модель, полученная на основе решения уравнения (1) методом Рэлея-Ритца.

Первая собственная частота колебаний ПУ рассчитывается по формуле:

$$
f_{\text {Пу }}=\frac{a}{2 k a^{2}} \sqrt{\frac{D}{m}} .
$$

Где $a-$ коэффициент, учитывающий характер закрепления платы;

$D$ - Цилиндрическая жесткость платы;

$m$ - Масса единицы площади Пу. 


$$
D=\frac{E S^{3}}{I 2\left(I-\mathrm{M}^{2}\right)^{\prime}}
$$

$$
m=ц S+m_{i} .
$$

$$
a=N_{1} \sqrt{N_{2}+N_{3}\left(\frac{a}{\mathrm{y}}\right)^{2}+N_{4}\left(\frac{a}{\mathrm{y}}\right)^{4}} .
$$

$$
a=18,33 \sqrt{\frac{5\left(1+2 \pi^{2} / 3+\pi^{4}\right) / 2+\mathrm{M}\left(1+\pi^{4}\right)}{1+3 \pi^{2}+21 \pi^{4} / 5+3 \pi^{\mathrm{n}}+\pi^{\mathrm{h}}}} ;
$$

$$
a \frac{13,66}{\pi} \frac{\sqrt{\left.1 / \pi^{2}\left(1-4 \pi^{2} / 3\right)+32 \pi^{4} / 3-4 \pi^{\Pi} / 3+\pi^{9}\right)+3 \mathrm{M}\left(1+22 \pi^{2}+\pi^{4}\right) / 8}}{1+11 \pi^{2} / 9+\pi^{4}}
$$

$$
\sigma=114,4 \sqrt{\frac{1+8 / 13 \pi^{4}+5 \mathrm{M} / 13}{1+7 \pi^{2}+119 \pi^{4} / 5+36 \pi^{6}+16 \pi^{и}}}
$$

$$
W_{j}(x, y)=\frac{a_{6} c_{k} x_{1}(x) Y_{1}(y)}{\left(2 \Pi \Gamma_{\Pi y}\right)^{2, \Gamma}}
$$

$$
\Gamma=\frac{I}{\sqrt{f \text { пу }}}
$$

$$
C_{k}=\frac{S_{1 k} S_{1 y}}{S_{2 x} S_{2 y}}
$$

Цилиндрическая жесткость $D$ и масса $m$ определяются по формулам (16), (17).

Где $E$ - усредненный модуль упругости и плотность материала платы соответственно;

$u$ - усредненный коэффициент Пуассона материала платы;

$S$ - Толщина платы; m- Масса РЭ, приходящаяся на единицу площади.

Для плат, закрепленных по контуру, коэффициент, а определится по формуле (18).

Где $a, b$ - длина и ширина плат соответственно;

$N_{1}, N_{2}, N_{3}, N_{4}$ - коэффициенты, приведенные для некоторых случаев в зависимости от способа крепления платы.
Для плат, закрепленных в четырех точках по углам, в пяти точках по углам и в центре, а также в шести точках по периметру коэффициент, а соответственно будет равен (19), (20), (21).

Где $a$ - отношение ширины платы b к длине a.

Амплитуда виброперемещения при резонансе, зависящая от соответствующих координат х и у (22).

Величина КМП приближенно определяется по формуле (23).

Коэффициент $C f c$ - определяется по формуле (24).

Где $S_{l x}, S_{l y}, S_{1 a x}, S_{a y}$ - интегралы, численные значения которых определяются для конкретного расчёта [5]. 


$$
X_{1}(x)=\sin \frac{\pi_{1} x}{a}+A_{1} \cos \frac{\pi_{1} x}{a}+B_{1} \operatorname{sh} \frac{\pi_{1} x}{a}+C_{1} \operatorname{ch} \frac{\pi_{1} x}{a}
$$

$$
Y_{1}(y)=\sin \frac{\pi_{1} y}{b}+A_{1} \cos \frac{\pi_{1} y}{b}+B_{1} \operatorname{sh} \frac{\pi_{1} y}{b}+C_{1} \operatorname{ch} \frac{\pi_{1} y}{b}
$$

$$
a_{j}(x, y)=a_{\text {б }} \sqrt{\left(\frac{C_{k} X_{1}(x) Y_{j}(y)}{\Gamma}\right)^{2}+1},
$$

$$
\mathrm{и}_{k}=\frac{\partial W_{1}\left(x_{1} y\right)}{\partial x} \frac{a_{b} C_{k} Y_{j}(y)}{\left(2_{k} f_{\text {Пу }}\right) \Gamma} \frac{\pi_{1}}{a}\left(\cos \frac{\pi_{1} x}{a}+A_{1} \sin \frac{\pi_{1} x}{a}+\mathrm{B}_{1} \operatorname{ch} \frac{\pi_{1} x}{a}+C_{1} \operatorname{ch} \frac{\pi_{1} x}{a}\right),
$$

$$
\mathrm{и}_{y}=\frac{\partial W_{1}\left(x_{1} y\right)}{\partial y} \frac{a_{b} C_{k} Y_{1}(y)}{\left(2_{k} f_{\text {Пу }}\right)^{a} \Gamma} \frac{\pi_{1}}{\mathrm{y}}\left(\cos \frac{\pi_{1} x}{\mathrm{y}}+A_{1} \sin \frac{\pi_{1} x}{\mathrm{y}}+\mathrm{B}_{1} \operatorname{ch} \frac{\pi_{1} x}{\mathrm{y}}+C_{1} \operatorname{ch} \frac{\pi_{1} x}{\mathrm{y}}\right),
$$

$$
\begin{aligned}
& \mathrm{y}_{1}=(x, y)=\frac{a_{6} C_{x} E S}{2\left(2 \Pi f_{\Pi \mathrm{Y}}\right)^{2} \Gamma\left(1-\mathrm{M}^{2}\right)} \\
&\left(-\sin \frac{\pi_{1} x}{a}-\right. A_{1} \cos \frac{\pi_{1} x}{a}+B_{1} \operatorname{ch} \frac{\pi_{1} x}{b}++C_{1} \operatorname{ch} \frac{\pi_{1} x}{b} \\
&\left.\quad+\mathrm{M}\left(-\sin \frac{\pi_{j} y}{b}-A_{j} \cos \frac{\pi_{j} y}{b}+B_{j} \operatorname{sh} \frac{\pi_{j} y}{b}+C_{j} \operatorname{ch} \frac{\pi_{j} y}{b}\right)\right),
\end{aligned}
$$

$$
\begin{aligned}
& \mathrm{y}_{2}=(x, y)
\end{aligned}
$$

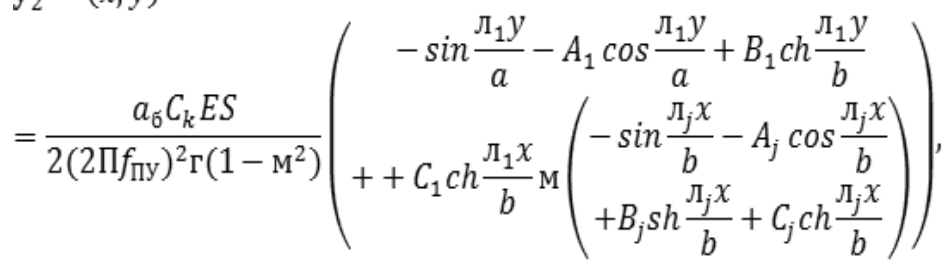

$$
\mathrm{y}(x, y)=\sqrt{\mathrm{y}_{1}\left(x_{1} y\right)^{2}+\mathrm{\Pi}_{2}\left(x_{1} y\right)^{2}-\mathrm{y}_{1} \mathrm{y}_{2}}
$$

Балочные функции $X_{l}(x), Y_{j}(y)$ определяются по формулам (25), (26).

Значения параметров $\pi_{l}, \pi_{j}$ и коэффициентов $A_{l}, A_{j}$ $B_{1}, B_{j}, C_{1}, C_{j}$, рассчитываются для конкретной платы [6].
Амплитуда виброускорения в различных точках платы рассчитывается по формуле (27).

Углы изгиба сечений ПУ вдоль осей X и Y соответственно определяются по формулам (28), (29), (30), (31), (32). 
Механические напряжения в точка с координатами х и у рассчитываются по формулам, полученным на основе выражений (9), (10), (11). (22), (25), (26)
Математический аппарат, приведенный в данном статье, успешно попользуется для экспресс-анализа на начальных стадиях проектирования печатных узлов.

\section{ЛИТЕРАТУРА}

1. Кофанов Ю.Н., Новиков А.С., Шалумов А.С. Информационная технология моделирования механических процессов в конструкциях радиоэлектронных средств. М.: Радио и связь, 2000.- 160 c.

2. Кофанов Ю.Н., Сотникова С.Ю. Информационные технологии теплового и механического моделирования радиоэлектронных средств. М.: НИУ ВШЭ, 2014. - $88 \mathrm{c}$.

3. Матвеев С.Е., Кофанов Ю. Н., Ройзман В.П. Методы системного анализа вибрационной прочности изделий. М.: Радио и связь, 2002. - 178 с.

4. Муромцев Д.Ю., Тюрин И. В., Белоусов О. А., Курносов Р. Ю. Проектирование функциональных узлов и модулей радиоэлектронных средств. Учебное пособие.- М: Издательство Лань СпБ, 2018. - 252 с.

5. Токарев М.Ф., Талицкий Е. Н., Фролов В.А. Механические воздействия и защита радиоэлектронной аппаратуры: Учеб. пособие для вузов/Под ред. В. А. Фролова. - М.: Радио и связь, 1984. - 224с.

6. Вибрации в технике: Справочник в 6-ти т.- Т. 6. /Под ред. К. В. Фролова.— М.: Машиностроение, 1981.— 456с.

○ Андрюхин Александр Гавриилович ( pr1110@list.ru ), Грачев Николай Николаевич ( nngrachev@mail.ru ),

Бойко Денис Владимирович ( den87-2004@mail.ru ).

Журнал «Современная наука: актуальные проблемы теории и практики»

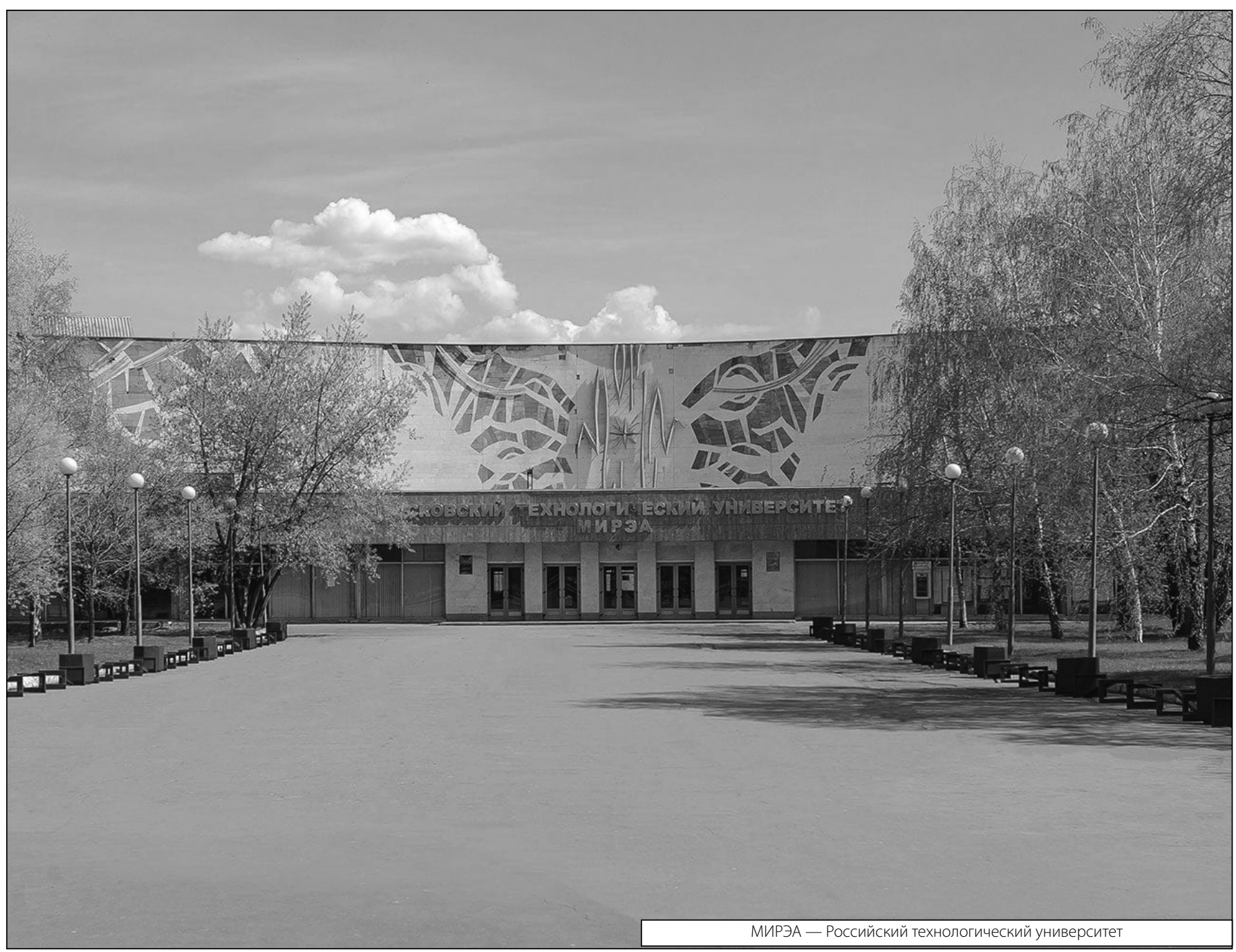

